\title{
Warmluftbefeuchtung unter CPAP mit und ohne Schlauchisolation
}

\author{
Heated Humidification during CPAP with and without Tube Insulation
}

Autoren

Institut
K.-H. Rühle, U. Domanski, M. Schröder, K. J. Franke, G. Nilius

HELIOS-Klinik Ambrock, Hagen, Universität Witten-Herdecke eingereicht 17.2.2010

akzeptiert 3.3.2010

Bibliografie

DOI http://dx.doi.org/

10.1055/s-0029-1244073

Online-Publikation: 12. 4. 2010

Pneumologie 2010; 64:

316-319 ๔ Georg Thieme

Verlag KG Stuttgart · New York ISSN 0934-8387

Korrespondenzadresse Prof. Dr. med. Karl-Heinz Rühle HELIOS-Klinik Ambrock Klinik für Pneumologie Ambrockerweg 60 58091 Hagen Klinik-Ambrock.Pneumo@ t-online.de

\section{Zusammenfassung \\ V}

Hintergrund: Patienten mit obstruktivem Schlafapnoesyndrom (OSAS) unter kontinuierlich positivem Überdruck (CPAP) klagen nicht selten über Austrocknung der Nasen- oder Rachenschleimhaut. Mit einem Warmluftbefeuchter (WLB) kann in vielen Fällen das Problem beseitigt werden. Vor allem bei kühler Umgebung bildet sich durch Abkühlung der Luft im Schlauch und in der Maske Kondenswasser. Um diese zu vermeiden, verwenden manche Patienten eine isolierende Schlauchhülle. Wir untersuchten den Einfluss der Umgebungstemperatur $\left(\mathrm{T}\right.$ in ${ }^{\circ} \mathrm{C}$ ) und relativen Luftfeuchtigkeit ( $\mathrm{rH}$ in \%) der Umgebung, des Beatmungsdrucks, einer Maskenleckage und Schlauchhülle auf die Temperatur und die Feuchtigkeit der Luft am Schlauchende beziehungsweise in der Maske bei OSAS-Patienten.

Methode: Alle Messungen erfolgten mit einem nicht regulierten WLB (S8, Fa. Resmed) und einem Temperatur- und Feuchtesensor (Fa. Testo, Lenzkirch).

Patienten: Untersucht wurden 8 Patienten mit OSAS während des Tages bei $16,4^{\circ} \mathrm{C}$.

Ergebnisse: Bei höherer Raumtemperatur stieg auch die Temperatur am Auslass des WLB an. Durch Isolation mit einer Schlauchhülle verringerte sich der Temperaturverlust im Schlauch um $2,3^{\circ} \mathrm{C}$. Während normaler Atmung fanden wir durch die Schlauchisolation abhängig vom Leckagefluss in der Maske eine mittlere Erhöhung der Temperatur zwischen 1,6 und $1,0^{\circ} \mathrm{C}$.

Schlussfolgerungen: Durch eine zusätzliche Isolierung mit einer Schlauchhülle kann, wenn auch geringfügig, die Maskentemperatur erhöht bzw. Kondenswasserbildung verringert werden.

\section{Abstract \\ $\nabla$}

Background: Patients with obstructive sleep apnoea syndrome (OSAS) under continuous positive pressure (CPAP) often complain about drying-up of the throat and nasal mucosa. In many cases the problem can be eliminated with a heated humidifier (WLB). Especially in a cold environment condensation forming on cooling of the air in the tube and the mask can be observed. To avoid this, some patients use an insulating tube covering. We investigated the effect of temperature $(\mathrm{T})$ and relative humidity $(\mathrm{rH})$ of the environment, the ventilation pressure, mask leaks, insulation of tubing on the $\mathrm{T}$ and $\mathrm{rH} \%$ of the delivered air at the end of the tube or in the mask in OSAS patients.

Method: All measurements were performed with a conventional WLB (S8, Resmed Fa) and a temperature and humidity sensor (Fa Testo, Lenzkirch).

Patients: 8 patients with OSAS were examined during the day at a room temperature of $16.4^{\circ} \mathrm{C}$.

Results: The temperature at the outlet of the WLB increased with a higher ambient temperature. Through isolation with a hose cover the temperature drop in the tube was reduced by $2.3^{\circ} \mathrm{C}$. By tube insulation a mean increase in temperature between 1.6 and $1.0 \mathrm{C}$ during normal breathing in dependence on the leakage flow in the mask was found.

Conclusions: Due to additional insulation with a tube cover the mask temperature can be increased, albeit slightly, and the formation of condensation is reduced. 


\section{Einleitung und Fragestellung \\ $\nabla$}

Patienten mit obstruktivem Schlafapnoesyndrom (OSAS), die mit kontinuierlich positivem Atemwegsdruck (CPAP) behandelt werden, klagen häufig über trockene Nasen-, Mund oder Rachenschleimhaut, sodass die Akzeptanz der Therapie beeinträchtigt ist [1 -3]. Diese Nebenwirkungen werden durch einen erhöhten Luftfluss durch Nase und Mund bei Mundleckagen ausgelöst [4]. Es wird deshalb empfohlen, die Luftfeuchtigkeit und Temperatur der zugeführten Luft durch Warmluftbefeuchtung (WLB) zu steigern, sodass die Reduktion der Luftfeuchtigkeit teilweise kompensiert werden kann [5]. Etwa 50\% der Patienten mit OSAS in Deutschland bevorzugen zum Schlafen eine Raumtemperatur von etwa $15^{\circ} \mathrm{C}$. Bei dieser geringen Raumtemperatur besteht die Gefahr, dass sich durch die Abkühlung Kondenswasser im Luftschlauch und in der Maske bildet und die Schlafqualität durch geringere absolute Luftfeuchtigkeit vermindert wird. Durch Beheizung des Schlauchs kann der Temperaturverlust verhindert werden, ohne dass der Taupunkt überschritten wird. Es konnte gezeigt werden, dass bei kühler Umgebung mittels geregelter WLB die Schlafqualität durch Reduktion der Wachzeit nach Schlafbeginn (WASO) deutlich verbessert wurde [6].

Um die Abkühlung der Luft und das Kältegefühl durch den Luftstrom zu verringern, verwenden manche Patienten eine Schlauchhülle, um höhere Temperaturen der zugeführten Luft zu erzielen. Diese Hülle ist auch kommerziell verfügbar: Schlauchhülle Nordkap. Fa. Heinen \& Löwenstein.

Unklar ist allerdings, ob und in welchem Ausmaß diese Maßnahme zu einer Temperaturzunahme führt.

Wir stellten uns deshalb die Frage, in welchem Ausmaß unter CPAP die Temperatur und die Luftfeuchtigkeit in der Maske beeinflusst wird. Insbesondere untersuchten wir den Einfluss

- der Temperatur und Luftfeuchtigkeit der Umgebung,

- des Beatmungsdrucks (höhere Flows infolge Auslassventil),

- einer simulierten Maskenleckage,

- einer Schlauchisolation.

\section{Methodik}

\section{Studie 1}

Untersucht wurde ein Warmluftbefeuchter mit offenem Regelkreis aus einer laufenden Serie (S8, Fa. Resmed). Der Drehschalter für Temperatur und Befeuchtung wurde auf Stufe drei gestellt. Temperatur $\left({ }^{\circ} \mathrm{C}\right)$ und relative Luftfeuchtigkeit $(\mathrm{rH} \%)$ wurden mit einer Frequenz von $0,5 \mathrm{~Hz}$ kontinuierlich aufgezeichnet. Erst nachdem sich ein steady state eingestellt hatte, wurde der Mittelwert über die nächsten zwei Minuten errechnet. Die absolute Luftfeuchtigkeit (mg/l) wurde nach der Formel von Chiumello errechnet [7].

Absolute Feuchtigkeit $\left(\mathrm{mgH}_{2} \mathrm{O} / \mathrm{l}\right)=$

relative Feuchtigkeit $\times\left(0,0387 \times \mathrm{T}^{2}-0,6066 \times \mathrm{T}+13,776\right)$

Bei den Ergebnissen handelt es sich jeweils um Einzelmessungen. Die Untersuchungen fanden in einem Raum des Schlaflabors im Januar statt, in einer Zeit, in der eine relativ geringe Luftfeuchtigkeit mit Außentemperaturwerten am Tage zwischen -4 und $+2^{\circ} \mathrm{C}$ herrschten. Konstante Raumbedingungen während der Untersuchung wurden durch Regulierung der Zimmertemperatur und gegebenenfalls Änderungen der Fensteröffnung erreicht.

Als Erstes bestimmten wir den Einfluss der Raumbedingungen auf die Befeuchterleistung. Dabei wurde der CPAP auf 8 mbar eingestellt und der Temperatur- und Feuchtigkeitssensor (Kombi-

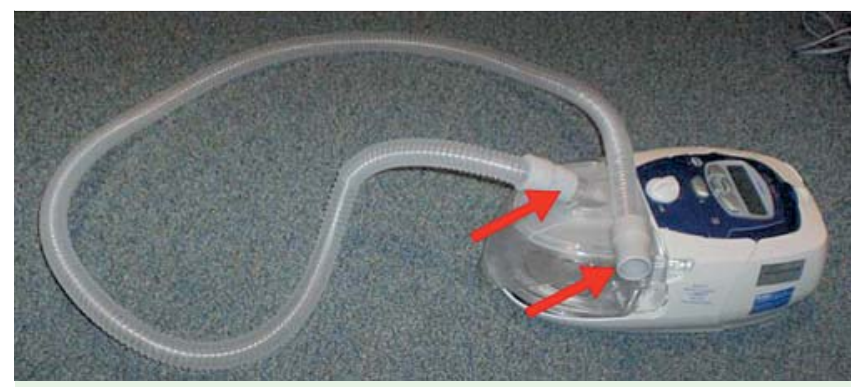

Abb. 1 Messpunkte zur Erfassung von Temperatur und Luftfeuchtigkeit am Befeuchter und am Schlauchende. Die Messung fand ohne Schlauchisolation statt. Temperatur des Raumes $16,4^{\circ} \mathrm{C}$.

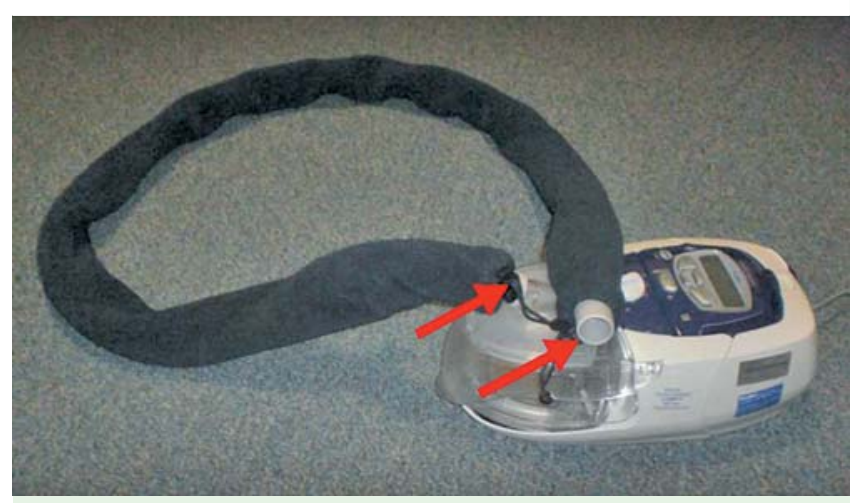

Abb. 2 Messung von Temperatur und Luftfeuchtigkeit am Befeuchter und am Schlauchende. Die Messung erfolgte unter Schlauchisolation mit einer Schlauchhülle. Temperatur des Raumes $16,4^{\circ} \mathrm{C}$.

nierter Miniatursensor für Temperatur und relativer Luftfeuchtigkeit, Fa. Testo, Lenzkirch/Germany) in der Öffnung des Warmluftbefeuchters angebracht. Aus Vorversuchen war uns bekannt, dass dadurch Flussraten $>80$ Liter pro Minute erreicht werden.

Bei einer Raumtemperatur von $16,4^{\circ} \mathrm{C}$ und $23,0^{\circ} \mathrm{C}$ wurden $\mathrm{rH} \%$ und Temperatur der ausströmenden Luft gemessen. Die Raumtemperatur wurde mit einem elektronischen Thermometer kontinuierlich registriert und schwankte um weniger als $1,0^{\circ} \mathrm{C}$. Unter denselben Bedingungen wurde der Sensor in das Schlauchende eingeführt und bei gleichem Luftfluss gemessen, sodass die Differenz zwischen der Luftfeuchtigkeit am Befeuchter und am Ende des Schlauches berechnet werden konnte (siehe $\bullet$ Abb. 1). Schließlich wurde der Schlauch mit einer Schlauchhülle aus Fleecestoff ummantelt und erneut Temperatur und $\mathrm{rH} \% \mathrm{am}$ Schlauchende gemessen (siehe $\bullet$ Abb. 2).

\section{Studie 2}

In der zweiten Studie wurde bei acht Patienten der Effekt einer Warmluftbefeuchtung auf die Luftfeuchtigkeit in der Maske mit und ohne Schlauchisolation untersucht $(\bullet$ Abb. 3). Wie in der ersten Studie handelt es sich um einen ungeregelten Warmluftbefeuchter. Die Messungen erfolgten unter einer Umgebungstemperatur von $16,4^{\circ} \mathrm{C}$. Die Patienten wurden jeweils mit demselben Maskentyp unter einem CPAP von 8 mbar, 12 mbar und 12 mbar mit zusätzlicher Leckage untersucht. In Vorversuchen hatten wir ermittelt, dass die CPAP-Druckerhöhung zu einer deutlichen Fluss-Steigerung führt. Die Flussraten wurden weiter erhöht durch das Anbringen einer zusätzlichen Leckage-Öffnung (siehe $\bullet$ Tab. 1). 


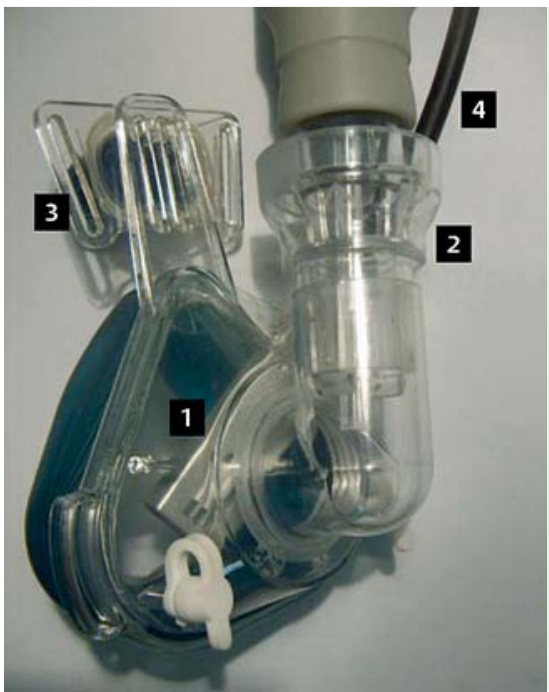

Abb. 3 Kombinierte Temperatur und Feuchtigkeitsmessung in einer CPAP-Maske. $1=$ Miniatursensor $2=$ Maskenport (Auslassventil). $3=$ Stirnpolster. 4 = Kabelzuführung .

Tab. 1 Druckabhängigkeit des Luftflusses unter CPAP. Mit zunehmendem CPAP-Niveau steigt der Fluss des Gerätes an. Durch Hinzufügen einer Öffnung wird ein zusätzlicher Leckagefluss erreicht.

$\begin{array}{llll} & \begin{array}{l}\text { Mit Auslass- } \\ \text { ventil allein } \\ \text { CPAP 8 mbar }\end{array} & \begin{array}{l}\text { Mit Auslass- } \\ \text { ventil allein } \\ \text { CPAP 12 mbar }\end{array} & \begin{array}{l}\text { Mit Auslass- } \\ \text { ventil + Leckage } \\ \text { CPAP 12 mbar }\end{array} \\ \text { Flow }(1 / \mathrm{min}) & 69 & 89 & 120\end{array}$

T und rH\% wurden über jeweils 10 Minuten kontinuierlich mit einer Frequenz von $0,5 \mathrm{~Hz}$ registriert. Aus den letzten 5 Minuten der Registrierung wurde der Mittelwert gebildet.

Patienten: Wir untersuchten acht Patienten mit obstruktivem Schlafapnoesyndrom in sitzender Position während des Tages zwischen 14:00 und 16:00 Uhr (mittleres Alter 51,8 \pm 8,8 Jahre, Größe 178,9 \pm 7,4 cm, Gewicht 102,4 $\pm 14,6$ kg, BMI 31,9 $\pm 4,0 \mathrm{~kg} /$ $\mathrm{m}^{2}$ ). Jeweils am Ende der Untersuchung wurden die Patienten über ihren subjektiven Eindruck und die Präferenz bezüglich der Schlauchisolation befragt. Die Grundsätze der Helsinki-Deklaration wurden beachtet.

Statistik: In den Tabellen werden die arithmetischen Mittelwerte mit Standardabweichung aufgeführt. Die Überprüfung auf signifikante Temperaturdifferenzen bei verbundenen Stichproben erfolgte mit dem nicht parametrischen Rang-Korrelations-Test nach Wilcoxon. Ein zweiseitiger p-Wert $<0,05$ wurde als signifikant angesehen. Die statistische Analyse wurde mit dem Statistikprogramm WinSTAT durchgeführt.

\section{Ergebnisse \\ $\nabla$}

\section{Studie 1}

Der Einfluss der Umgebungsbedingungen auf die Temperatur und $\mathrm{rH} \%$ des Befeuchters war deutlich messbar. Bei höherer Raumtemperatur stieg die Temperatur am Auslass des Befeuchters um $8,3^{\circ} \mathrm{C}$ an, die $\mathrm{rH} \%$ fiel ab, die Berechnung der aH ergab eine Zunahme von 2,5 mg/l Luft (siehe $\bullet$ Tab. 2).

Ohne Schlauchisolation fiel die Temperatur auf der Strecke vom Befeuchter bis zum Schlauchende um $4,3^{\circ} \mathrm{C}$ ab. Die absolute Luftfeuchtigkeit blieb, wie zu erwarten war, konstant (siehe $\bullet$ Tab. 3). Im Vergleich zur Temperaturabnahme auf $19,4^{\circ} \mathrm{C}$ ohne Isolation führte die Schlauchisolation zu einer geringeren Abnahme der
Tab. 2 Abhängigkeit der Luftfeuchtigkeit und Temperatur am Warmluftbefeuchter von der Raumtemperatur.

\begin{tabular}{|c|c|c|c|}
\hline & $\begin{array}{l}\text { Raum- } \\
\text { temperatur } \\
16,4^{\circ} \mathrm{C}\end{array}$ & $\begin{array}{l}\text { Raum- } \\
\text { temperatur } \\
23,0^{\circ} \mathrm{C}\end{array}$ & Differenz \\
\hline $\mathrm{T}\left({ }^{\circ} \mathrm{C}\right)$ & 23,7 & 32,0 & $+8,3$ \\
\hline $\mathrm{rH} \%$ & 44,5 & 34,9 & $-9,6$ \\
\hline $\mathrm{aH}$ (mgH $\left.\mathrm{m}_{2} \mathrm{O} / \mathrm{luft}\right)$ & 9,4 & 11,9 & $+2,5$ \\
\hline
\end{tabular}

Tab. 3 Vergleich der T und rH\% am Befeuchter und am Schlauchende (ohne Isolation) bei einer Raumtemperatur von $16,4^{\circ} \mathrm{C}$. Temperaturverlust von $4{ }^{\circ} \mathrm{C}$ im Atemschlauch. Die relative Luftfeuchtigkeit nimmt zu, die absolute Luftfeuchtigkeit bleibt praktisch konstant.

\begin{tabular}{|lrll} 
& $\mathbf{T}\left({ }^{\circ} \mathbf{C}\right)$ & $\mathbf{r H \%}$ & $\begin{array}{l}\mathbf{a H} \\
\left(\mathbf{m g H} \mathbf{H}_{\mathbf{2}} \mathbf{O} / \mathbf{l} \text { Luft) }\right.\end{array}$ \\
& & & 9,4 \\
\hline direkt am Befeuchter & 23,7 & 44,5 & 9,9 \\
\hline Schlauchende & 19,4 & 59,8 & 0,5 \\
\hline Differenz & $-4,3$ & $+15,3$ & \\
\hline
\end{tabular}

Tab. 4 Vergleich der T und rH\% am Schlauchende ohne und mit Schlauchisolation ( 8 mbar, Fluss $>80 \mathrm{l} / \mathrm{min}$, Raumtemperatur $16,4^{\circ} \mathrm{C}$ ). Mit Isolation steigt die Temperatur um $2,3^{\circ} \mathrm{C}$ an, $\mathrm{rH} \%$ fällt um $10 \%$ ab. Die absolute Luftfeuchtigkeit bleibt praktisch konstant.

\begin{tabular}{|llll} 
& $\begin{array}{l}\text { Temperatur } \\
\left({ }^{\circ} \mathbf{C}\right)\end{array}$ & $\mathbf{r H \%}$ & $\begin{array}{l}\mathbf{a H} \\
(\mathbf{m g H} \mathbf{2} \mathbf{O} \mathbf{l} \text { Luft) }\end{array}$ \\
\hline ohne Isolation & 19,4 & 59,8 & 9,9 \\
\hline mit Isolation & 21,7 & 50,0 & 9,4 \\
\hline Differenz & 2,3 & $-9,8$ & 0,5 \\
\hline
\end{tabular}

Temperatur auf lediglich $21,7^{\circ} \mathrm{C}$. Bei praktisch konstanter absoluter Luftfeuchtigkeit fiel die rH\% um etwa $10 \%$ (siehe $\bullet$ Tab. 4).

\section{Studie 2}

Abhängig von der Höhe von CPAP fiel die absolute Luftfeuchtigkeit insbesondere bei simulierter Leckage deutlich ab. Bei Schlauchisolation stieg die Temperatur in der Maske im Vergleich zum Schlauch ohne Schlauchhülle um 1,6 beziehungsweise 1,1 und $1,0^{\circ} \mathrm{C}$ an. Wegen der Beziehung zwischen Temperatur und rH\% fiel die relative Luftfeuchtigkeit zwischen 6 und 10\%. Die absolute Luftfeuchtigkeit änderte sich praktisch nicht (siehe - Tab. 5).

\section{Diskussion}

Wir konnten nachweisen, dass unter CPAP-Therapie mit Warmluftbefeuchtung in einer kühlen Schlafraumumgebung durch Isolation des zuführenden Schlauches, verglichen mit der normalen Schlauchzuführung, die Temperatur in der Maske beim atmenden Patienten zwischen $1-2^{\circ} \mathrm{C}$ angehoben werden kann. Noch deutlicher wird dieser Effekt mit $>2{ }^{\circ} \mathrm{C}$ Temperaturdifferenz, wenn man unter hohen Flussraten den Effekt der Schlauchhülle auf der Strecke vom Befeuchter bis zur Schlauchmündung an der Maske erfasst.

Unklar bleibt, ob die Erhöhung der Temperatur dem Patienten einen subjektiven Profit bringt. In unserer Befragung nach Beendigung der nicht verblindeten Untersuchung bevorzugten die meisten Patienten die CPAP-Therapie mit Isolation des Schlauches durch eine Schlauchhülle (6 von 8 Patienten). 


\begin{tabular}{|c|c|c|c|c|c|c|c|c|}
\hline & \multicolumn{3}{|l|}{$\mathrm{T}\left({ }^{\circ} \mathrm{C}\right)$} & \multicolumn{3}{|l|}{$\mathrm{rH} \%$} & \multicolumn{2}{|c|}{$\mathrm{aH}$ ( $\left.\mathrm{mgH}_{2} \mathrm{O} / \mathrm{I} \mathrm{Luft}\right)$} \\
\hline & ohne & mit & Diff & ohne & mit & Diff & ohne & mit \\
\hline $8 \mathrm{mbar}$ & $\begin{array}{l}19,7 \\
(3,0)\end{array}$ & $\begin{array}{l}21,3 \\
(2,8)\end{array}$ & 1,6 & $\begin{array}{l}95,3 \\
(5,1)\end{array}$ & $\begin{array}{l}89,1 \\
(11,1)\end{array}$ & $-6,2$ & $\begin{array}{l}16,4 \\
(3,2)\end{array}$ & $\begin{array}{l}16,8 \\
(4,1)\end{array}$ \\
\hline $12 \mathrm{mbar}$ & $\begin{array}{l}19,9 \\
(2,5)\end{array}$ & $\begin{array}{l}21,0 \\
(2,2)\end{array}$ & 1,1 & $\begin{array}{l}94,7 \\
(5,8)\end{array}$ & $\begin{array}{l}87,5 \\
(9,5)\end{array}$ & $-7,2$ & $\begin{array}{l}16,3 \\
(2,8)\end{array}$ & $\begin{array}{l}16,1 \\
(3,1)\end{array}$ \\
\hline 12 mbar + Leck & $\begin{array}{l}20,1 \\
(1,8)\end{array}$ & $\begin{array}{l}21,1 \\
(1,5)\end{array}$ & 1,0 & $\begin{array}{l}85,2 \\
(9,9)\end{array}$ & $\begin{array}{l}75,6 \\
(11,1)\end{array}$ & $-9,6$ & $\begin{array}{l}14,7 \\
(1,5)\end{array}$ & $\begin{array}{l}13,8 \\
(2,1)\end{array}$ \\
\hline
\end{tabular}

Tab. 5 Temperatur, relative und absolute Luftfeuchtigkeit in der Maske von acht Patienten ohne und mit Schlauchisolation mit OSAS.

Mittelwert und in Klammern die Standardabweichung. Raumtemperatur und Luftfeuchtigkeit: $16,0 \pm 1,0{ }^{\circ} \mathrm{C}$, $\mathrm{rH} \% 33,7$ $\pm 7,0$, aH 4,7 $\pm 1,2 \mathrm{mgH}_{2} \mathrm{O} / \mathrm{I}$. Im Vergleich zur Therapie ohne Schlauchhülle fand sich unter Schlauchisolation eine signifikant höhere Maskentemperatur bei CPAP 8,12 mbar und CPAP 12 mbar mit simulierter Leckage $(p<0,05)$.

Wenn es gelingt, den Temperaturverlust im Schlauch zu verringern, kann die Kondensation des Wasserdampfes im Schlauch bis zu einem bestimmten Grade verhindert werden, sodass eine höhere absolute Luftfeuchtigkeit mit geringerer Austrocknung der Schleimhäute erzielt werden kann.

In kalter Umgebung kühlt die Luft im Schlauch relativ deutlich ab. Der Effekt dieser Abkühlung sei an einem Beispiel gezeigt: Bei einer Temperatur von $27,8^{\circ} \mathrm{C}$ und $90 \%$ Luftfeuchtigkeit liegt die absolute Luftfeuchtigkeit bei $24,1 \mathrm{mg} / \mathrm{L}$. Bei einem Temperaturverlust im Schlauch von 1,8 Grad Celsius fällt die Temperatur auf $26^{\circ} \mathrm{C}$, die Luftfeuchtigkeit steigt dabei auf 100 Prozent an, die absolute Luftfeuchtigkeit liegt weiterhin bei $24,1 \mathrm{mg} / \mathrm{l}$. Wenn die Temperatur im Schlauch weiter sinkt, wird der Taupunkt überschritten und das Wasser kondensiert im Schlauch oder in der Maske, da die rH\% nicht über $100 \%$ ansteigen kann.

Wie wir in dem Vorversuch zeigen konnten, beeinflusst die Temperatur und relative Luftfeuchtigkeit im Raum das Ergebnis der Warmluftbefeuchtung erheblich, da der Warmluftbefeuchter der angesaugten Luft lediglich eine bestimmte Menge Wasserdampf und Temperatur hinzufügen kann. Vor allem bei einer Umgebungstemperatur im Schlafraum von etwa $16^{\circ} \mathrm{C}$ wird dieser Einfluss relevant.

Zunehmende Beatmungsdrücke führen zu höheren Flussraten und damit bei fehlender Regelung zu einem Absinken der Luftfeuchtigkeit am Schlauchende und in der Maske. So konnte bei einem Vergleich von Warmluftbefeuchtern ein Absinken der Luftfeuchtigkeit zum Teil auf $<80 \%$ festgestellt werden [8]. Auch wir stellten durch direkte Messungen in der Maske eine Abhängigkeit der Luftfeuchtigkeit von dem vom Gerät gelieferten Luftfluss fest. Neuere CPAP-Geräte berücksichtigen die Umgebungstemperatur, die aktuelle Luftfeuchtigkeit im Raum und den Fluss des Gerätes. Durch Heizung des Schlauches kann die Kondensation von Wasserdampf unter Berücksichtigung aller Kenngrößen vermieden werden.

Generell sollte festgehalten werden, dass die oberen Atemwege, vor allem die Nasenschleimhaut, normalerweise eine hohe Befeuchterkapazität besitzen, sodass die Differenz der Luftfeuchtigkeit zwischen der eingeatmeten Luft und dem distalen Drittel der Trachea (die aH liegt hier bei $44,3 \mathrm{mg} / \mathrm{l}$ ) ausgeglichen werden kann [9]. Allerdings kann unter CPAP bei Mundatmung und hohen Flüssen der nasale Widerstand durch Anschwellen der Nasenschleimhaut infolge Irritationen durch nicht ausreichend angewärmte und angefeuchtete Luft erheblich ansteigen [10]. Unklar bleibt, bis zu welchen Schwellenwerten die Temperatur und relative Luftfeuchtigkeit absinken muss, bis nasale Beschwerden und Rachensymptome erscheinen.

Zusammenfassend können wir feststellen, dass in kühlen Umgebungen durch einfache Schlauchisolation die Abkühlung der Luft bis zur Schlauchmündung in der Maske vermindert wird. Die Patienten profitieren eventuell durch die leichte Zunahme der Temperatur in der Maske durch die zusätzliche Isolation des Schlauches. Weiterhin ist es möglich, am Warmluftbefeuchter die Heizleistung etwas zu erhöhen, da der Taupunkt verschoben wird und damit die sonst stattfindende Kondensation von Wassertröpfchen im Schlauch vermieden wird. Allerdings ist der Effekt nicht sehr ausgeprägt. Durch den Einsatz von geregelten Warmluftbefeuchtern mit Schlauchheizung kann die absolute Luftfeuchtigkeit deutlich stärker angehoben werden.

\section{Interessenkonflikte \\ $\nabla$}

K. H. Rühle und G. Nilius erhielten Studiengelder von Fisher \& Paykel Healthcare, Heinen und Löwenstein, ResMed und Weinmann. Diese Gelder gingen in wissenschaftliche Projekte der Klinik. Die aktuelle Originalarbeit wurde von der HELIOS Forschungsförderung unterstützt. Die Präsentation des Themas ist unabhängig und die Darstellung des Inhalts produktneutral.

\section{Literatur}

1 Pépin JL, Leger P, Veale $D$ et al. Side effects of nasal continuous positive airway pressure in sleep apnea syndrome. Study of 193 patients in two French sleep centers. Chest 1995; 107: 375-381

2 Bachour A, Maasilta P. Mouth breathing compromises adherence to nasal continuous positive airway pressure therapy. Chest 2004; 126 : $1248-1254$

3 Mador MJ, Krauza M, Pervez A et al. Effect of heated humidification on compliance and quality of life in patients with sleep apnea using nasal continuous positive airway pressure. Chest 2005; 128: 2151 - 2158

4 Ruhle KH, Nilius G. Mouth breathing in obstructive sleep apnea prior to and during nasal continuous positive airway pressure. Respiration 2008; 76: 40-45

5 Martins De Araújo MT, Vieira SB, Vasquez EC et al. Heated humidification or face mask to prevent upper airway dryness during continuous positive airway pressure therapy. Chest 2000; 117: 142 - 147

6 Nilius G, Domanski U, Franke KJ et al. Impact of a controlled heated breathing tube humidifier on sleep quality during CPAP therapy in a cool sleeping environment. Eur Respir J 2008; 31: 830-836

7 Chiumello D, Chierichetti $M$, Tallarini $F$ et al. Effect of a heated humidifier during continuous positive airway pressure delivered by a helmet. Crit Care 2008; 12: R55

8 Rouadi P, Baroody FM, Abbott D et al. A technique to measure the ability of the human nose to warm and humidify air. J Appl Physiol 1999; 87: 400-406

9 Wenzel M, Wenzel G, Klauke M et al. Characteristics of several humidifiers for CPAP-therapy, invasive and non-invasive ventilation and oxygen therapy under standardised climatic conditions in a climatic chamber. Pneumologie 2008; 62: 324-329

10 Richards GN, Cistulli PA, Ungar RG et al. Mouth leak with nasal continuous positive airway pressure increases nasal airway resistance. Am Respir Crit Care Med 1996; 154: 182-186 